Bio - grafía. Escritos sobre la Biología y su Enseñanza. ISSN 2027-1034

Edición Extraordinaria. p.p. 1533 - 1540

Memorias del IX Encuentro Nacional de Experiencias en Enseñanza de la Biología y la Educación Ambiental. IV Congreso Nacional de Investigación en Enseñanza de la Biología.

\title{
ESTUDIO DE CASO COMO ESTRATEGIA DIDÁCTICA PARA EL PROCESO ENSEÑANZA-APRENDIZAJE: RETOS Y OPORTUNIDADES
}

\author{
María Cristina Gamboa Mora'
}

\begin{abstract}
RESUMEN
El estudio de caso como estrategia didáctica, se implementó en la Universidad de Harvard, Cambridge-Massachusetts en el programa de Derecho, hacia 1914 y como método se cristalizó hacia 1935. Como estrategia docente para diversas disciplinas, se ha extendido a través del tiempo. Los casos se pueden configurar como: centrados en las descripciones, centrados en el análisis crítico para la toma de decisiones y casos para la resolución de problemas. Con base en la propuesta del Instituto Tecnológico de Monterrey sobre el estudio de caso como estrategia didáctica, se establece que el estudio de caso tiene tres fases que se denominan: fase de identificación, fase de toma de decisiones y fase de resolución de problemas y se establecen subfases para la implementación en el ambiente de aprendizaje. Las estrategias didácticas son un conjunto de acciones que suscitan procesos de pensamiento que propenden por la adquisición de habilidades como precursoras de las competencias que son las capacidades que se procuran para un desempeño óptimo y que, responden a una necesidad de conocimiento en condiciones de calidad. El taller pretende generar un ambiente de enseñanza-aprendizaje vivencial sobre las oportunidades de construcción de conocimiento que puede emerger del análisis de un caso, con el fin de adquirir competencia para la implementación de la estrategia en otros espacios educativos arguiendo a la responsabilidad personal y social del docente.
\end{abstract}

Palabras clave: estrategia didáctica, ambiente de enseñanza-aprendizaje, estudio de caso, pedagogía y didáctica.

\section{CASE STUDY AS A DID ACTIC STRATEGY FOR THE TEACHING-LEARNING PROCESS: CHALLENGES AND OPPORTUNITIES ABSTRACT}

The case study as a didactic strategy was implemented at Harvard University, Cambridge-Massachusetts in the Law program, toward 1914 and as a method it was stated in around the 1935. As a teaching strategy for various disciplines, it has spread over

\footnotetext{
${ }^{1}$ Doctora en Innovación e investigación en Didáctica. Docente Catedrática del Programa Curricular de Licenciatura en Biología, PCLB-Universidad Distrital Francisco José de Caldas y Docente Asociado Universidad Nacional Abierta y a Distancia. Líder del grupo de Investigación interinstitucional UNAD-UD: "Ambientes de Enseñanza-Aprendizaje de las Ciencias Básicas (AMECI)”. Correo electrónico: maria.gamboa@unad.edu.co o mcgamboam@udistrital.edu.co
} 


\title{
Bio - grafía. Escritos sobre la Biología y su Enseñanza. ISSN 2027-1034
}

\section{Edición Extraordinaria. p.p. 1533 - 1540}

\author{
Memorias del IX Encuentro Nacional de Experiencias en Enseñanza de la Biología y la \\ Educación Ambiental. IV Congreso Nacional de Investigación en Enseñanza de la \\ Biología.
}

time. Cases can be configured as: focused on descriptions, focused on critical analysis for decision making and problem solving cases. Based on the proposal of the Technological Institute of Monterrey on the case study as a didactic strategy, it is established that the case study has three phases that are called: identification phase, decision making phase and problem solving phase; and there are sub-phases for implementation in the learning environment. The didactic strategies are a set of actions that stimulate thought processes that tend to the acquisition of skills as precursors of the competences that are the capacities that are sought for an optimal performance and wich respond to a need of knowledge in high quality conditions. The workshop aims to generate a teaching-learning environment about the opportunities for knowledge construction that can emerge from the analysis of a case, in order to acquire competence for the implementation of the strategy in other educational spaces, arguing for personal and social responsibility of the teacher.

KEYWORDS: didactic strategy, teaching-learning environment, case study, pedagogy and didactics.

\section{INTRODUCCIÓN}

El Método del Caso (MdC), denominado también análisis o estudio de casos, como técnica de aprendizaje tuvo su origen en la Universidad de Harvard (1914), con el fin de que los estudiantes de Derecho, en el aprendizaje de las leyes, se enfrentaran a situaciones reales o casos de la vida adaptados a la enseñanza y tuvieran que tomar decisiones, valorar actuaciones, emitir juicios fundamentados, entre otras. El MdC fue extendiéndose a otros contextos y se ha convertido en una estrategia muy eficaz para que los estudiantes adquieran diversos aprendizajes y desarrollen diferentes habilidades porque el actor es protagonista en la resolución de los casos. (Bruner 1960) señala que el método del caso, anima al alumno a hacer preguntas y formular sus propias respuestas. (Como se cita en Castro, 2014). Otros como Wassermann (1994) afirma que "Ios buenos casos se construyen en torno a problemas o "grandes ideas", es decir, aspectos significativos de una materia o asunto que garantizan un examen serio y a profundidad. Las narrativas se estructuran por lo general a partir de problemas y personas de la vida real. (Como se cita en Díaz-Barriga, 2005, p.16).

Ahora bien para la implementación de una estrategia didáctica, los ambientes de enseñanza-aprendizaje propicios son aquellos que surgen con una interacción diversa, en la cual prevalece el diálogo como vía óptima para establecer relaciones de igualdad y no de poder. Bajo estas condiciones emerge el reconocimiento por el otro, a través de la escucha y la participación mediada por acciones como la lectura, la escritura, entre otras. (Álvarez, González y Larrinaga, 2013). En ese ambiente, la planeación pedagógicodidáctica toma un lugar preponderante al requerir de la definición de dos aspectos relevantes para el proceso educativo: la estrategia pedagógica y la estrategia didáctica para el logro de los objetivos. En un ambiente escolarizado el propósito anterior, puede asociarse directamente a la consecución de competencias, que desde la concepción de 
Bio - grafía. Escritos sobre la Biología y su Enseñanza. ISSN 2027-1034

Edición Extraordinaria. p.p. 1533 - 1540

Memorias del IX Encuentro Nacional de Experiencias en Enseñanza de la Biología y la

Educación Ambiental. IV Congreso Nacional de Investigación en Enseñanza de la Biología.

Tobón (2006) se asocia a "un proceso complejo de desempeño con idoneidad en un determinado contexto, con responsabilidad" (p.6).

El concepto de competencia se empezó a estructurar en los años 70 con base en las aportaciones de Chomsky y Skinner. Chomsky (1970) propuso el concepto de competencia lingüística como una estructura mental implícita y genéticamente determinada que se pone en acción mediante el desempeño comunicativo, involucrando competencia con desempeño y asociándola a algo interno. Por su parte, Skinner asocia el concepto de competencia al comportamiento, observable, efectivo y verificable. Vygotsky (1985) y Brunner (1992) coinciden en señalar que las competencias son acciones que se dan a partir de la mente y ella, se construye en relaciones sociales, y se actualiza por la cultura. (Como se cita en Tobón, 2006).

Otro elemento crucial es el docente como ejecutor idóneo del acto didáctico (ver Figura 1), que debe propender por el aprendizaje de los estudiantes planificando estrategias pedagógico-didácticas que permeen el desarrollo humano cualificado. La estrategia pedagógica o las estrategias pedagógicas ha (n) de propender por el desarrollo de la persona, dirigida a un objetivo u objetivos, y la estrategia didáctica o las estrategias didácticas procura (n) por la promoción de los procesos cognitivos con el objetivo de aprender y construir conocimiento contextualizado y cualificado.

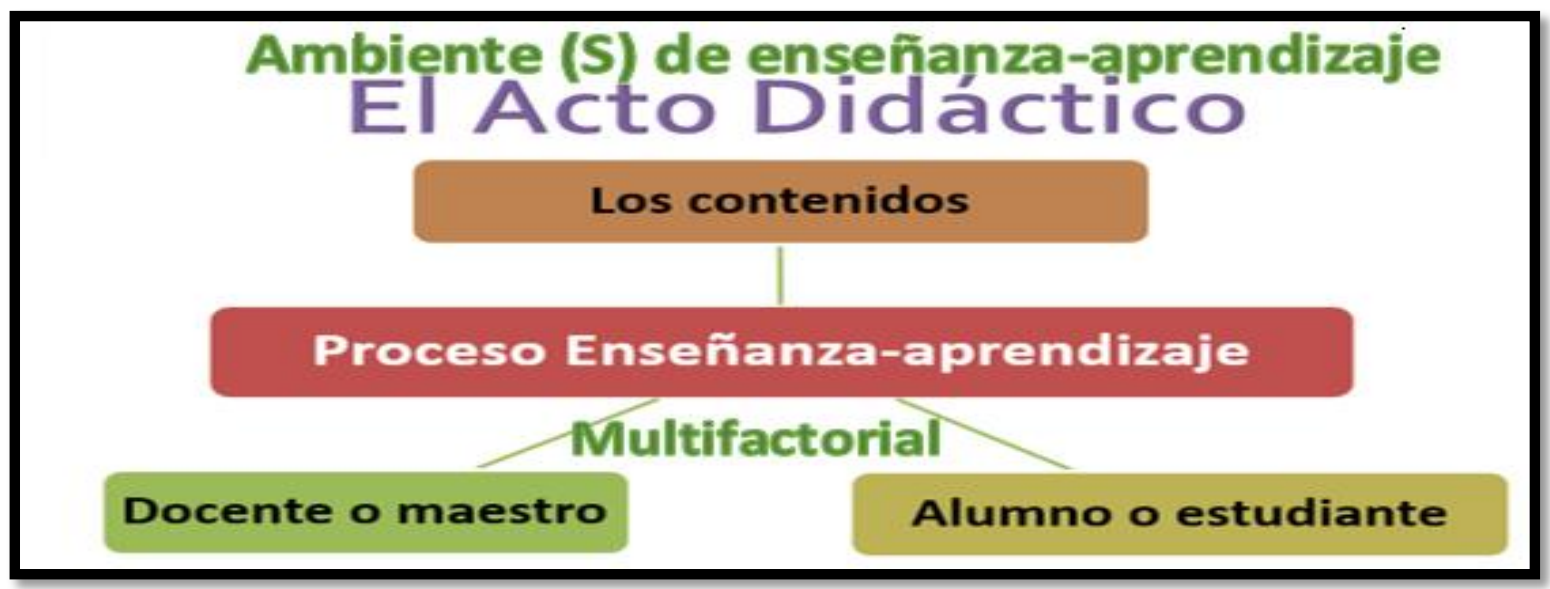

\section{llustración 1}

Figura 1. El acto didáctico. Nota: Elaboración propia.

Consecuentemente, el acto educativo y el proceso educativo, son complejos, y la pedagogía que para muchos hasta hace poco era considerada como la única ciencia de la educación, no aborda de manera integral, el proceso. La definición del objeto material de la didáctica y el objeto formal de la misma, le han permitido un estatus como ciencia de la educación. Díaz Alcaraz (2002) señaló que situar a la didáctica en el estatus de ciencias de la educación, es apropiado, por cuanto que al definir el objeto material de la misma, como el estudio del proceso instructivo, es viable identificar el aporte que esta ciencia 
Bio - grafía. Escritos sobre la Biología y su Enseñanza. ISSN 2027-1034

Edición Extraordinaria. p.p. 1533 - 1540

Memorias del IX Encuentro Nacional de Experiencias en Enseñanza de la Biología y la

Educación Ambiental. IV Congreso Nacional de Investigación en Enseñanza de la Biología.

hace a la educación. Por su parte Valdes y Valdes (2004), afirman que la Didáctica, ha pasado a la elaboración de un cuerpo de conocimientos propios, donde convergen concepciones filosóficas, la epistemología e historia de la ciencia, la psicología y el conocimiento de las ciencias.

Es por lo anterior, que la pedagogía y la didáctica establecen lazos desde los cuales se puede comprender el que hacer educativo, como menciona Zambrano: "la didáctica es para la pedagogía su mejor aliado, ella le aporta los elementos de acción práctica para que el pedagogo pueda actuar y ejercer de mejor forma sus actividades en el espacio de la enseñanza y el aprendizaje" (2002, p. 32). En este momento, cobra sentido abordar concretamente las concepciones que sobre las estrategias didácticas como las promotoras de los procesos cognitivos se han promulgado. Pozo (1990) afirma que son un conjunto de operaciones mentales que en secuencias o procedimientos se integran con el propósito de facilitar la adquisición, almacenamiento o utilización de la información. Monés (2006) por su parte, las concibe como estructuras de actividad que permean los objetivos y contenidos por lo cual las considera análogas a las técnicas en concordancia con el Instituto Tecnológico de Monterrey (2005) al definirlas como el conjunto de procedimientos, apoyados en técnicas de enseñanza que tienen como objetivo a través de actividades lograr el aprendizaje. (Mansilla y Beltrán, 2013). Para el grupo de investigación Ambientes de enseñanza-aprendizaje de las Ciencias Básicas, AMECI. Las estrategias didácticas son un conjunto de acciones que suscitan procesos de pensamiento que propenden por la adquisición de habilidades como precursoras de las competencias.

Finalmente, y con base en la propuesta del Instituto Tecnológico de Monterrey (s.f) sobre el estudio de caso como estrategia didáctica, se establecen tres fases que se denominan: fase de identificación, fase de toma de decisiones y fase de resolución de problemas. Y se propone para su implementación, subfases que configuran a su vez: casos centrados en el estudio de las descripciones, casos centrados en el análisis crítico para la toma de decisiones y casos para la resolución de problemas, las fases y las acciones establecidas para la resolución de un caso, se presentan en la tabla 1.

Tabla 1. Propuesta para la implementación del estudio de caso como estrategia didáctica

\begin{tabular}{|l|l|l|}
\hline \multicolumn{2}{|c|}{ Estudio de Caso } \\
\hline Fases & \multicolumn{1}{|c|}{ Procesos involucrados } & $\begin{array}{c}\text { Denominación } \\
\text { del Caso }\end{array}$ \\
\hline & $\begin{array}{l}\text { Lectura y estudio de los casos presentados para la } \\
\text { toma de conciencia sobre la situación descrita en } \\
\text { cada uno de ellos. }\end{array}$ & \\
& $\begin{array}{l}\text { Subfase preliminar } \\
\text { Reportar los elementos descriptivos principales } \\
\text { involucrados. }\end{array}$ & $\begin{array}{l}\text { Casos centrados } \\
\text { en el estudio de } \\
\text { las } \\
\text { Subfase de expresión }\end{array}$ \\
\hline
\end{tabular}


Bio - grafía. Escritos sobre la Biología y su Enseñanza. ISSN 2027-1034

Edición Extraordinaria. p.p. 1533 - 1540

Memorias del IX Encuentro Nacional de Experiencias en Enseñanza de la Biología y la Educación Ambiental. IV Congreso Nacional de Investigación en Enseñanza de la Biología.

\begin{tabular}{|c|c|c|}
\hline $\begin{array}{l}\text { Fase de } \\
\text { identificación }\end{array}$ & $\begin{array}{l}\text { Análisis a través de la puesta en común de las } \\
\text { situaciones descritas en el caso en búsqueda de } \\
\text { sentido de la información reportada. } \\
\text { Subfase de contraste } \\
\text { Planteamiento de hipótesis sobre el caso que } \\
\text { aproximan al estudio de diversas ópticas de } \\
\text { explicación y análisis de las situaciones. } \\
\text { Subfase de reflexión teórica } \\
\text { Análisis de los conceptos teóricos que se requieren } \\
\text { para la comprensión del tema en el cual se centra la } \\
\text { situación. }\end{array}$ & $\begin{array}{l}\text { Procesos } \\
\text { cognitivos } \\
\text { involucrados: } \\
\text { Análisis, síntesis } \\
\text { y pensamiento } \\
\text { crítico. }\end{array}$ \\
\hline $\begin{array}{l}\text { Fase de toma } \\
\text { de } \\
\text { decisiones }\end{array}$ & $\begin{array}{l}\text { Revisión del contexto en cada caso y variables que } \\
\text { se describen en cada situación. } \\
\text { Subfase estudiar y analizar } \\
\text { Reconocer y localizar información adicional para } \\
\text { comprender los casos. Jerarquizar los problemas de } \\
\text { acuerdo con la importancia en el contexto estudiado. } \\
\text { Subfase identificar y detectar } \\
\text { Alternativas de acción para lograr cada uno de los } \\
\text { cambios requeridos para solucionar las situaciones. } \\
\text { (Plantear alternativas de acción). } \\
\text { Sub fase estudiar y generar } \\
\text { Reflexionar sobre las decisiones tomadas y sus } \\
\text { implicaciones para seleccionar las que impliquen } \\
\text { menos dificultades e impactos negativos, } \\
\text { estableciendo cuáles son las estrategias para } \\
\text { implementar de manera óptima la decisión tomada. } \\
\text { Estudiar e implementar }\end{array}$ & $\begin{array}{l}\text { Casos centrados } \\
\text { en el análisis } \\
\text { crítico de toma } \\
\text { de decisiones } \\
\text { Procesos } \\
\text { cognitivos } \\
\text { involucrados: } \\
\text { Análisis, síntesis } \\
\text { y pensamiento } \\
\text { crítico }\end{array}$ \\
\hline $\begin{array}{l}\text { Fase de } \\
\text { propuestas }\end{array}$ & $\begin{array}{l}\text { De manera individual con base en el caso objeto de } \\
\text { estudio, debe establecer las situaciones } \\
\text { problemáticas que están involucradas en el caso } \\
\text { socializado y escalonarlas de acuerdo con la } \\
\text { importancia que representan en el contexto } \\
\text { estudiado. } \\
\text { Subfase de identificación y jerarquización } \\
\text { Individualmente cada participante estudia la toma de } \\
\text { decisiones descrita en el relato presentado, se } \\
\text { registran las apreciaciones y emite su opinión sobre } \\
\text { el proceso seguido teniendo en cuenta las } \\
\text { consecuencias tomadas al respecto. Es necesario } \\
\text { valorar las actuaciones de cada uno de los actores } \\
\text { involucrados en el caso objeto de estudio } \\
\text { Subfase de análisis crítico y toma de decisiones } \\
\text { El equipo valorará las acciones tomadas y las }\end{array}$ & $\begin{array}{l}\text { Casos centrados } \\
\text { la resolución de } \\
\text { problemas } \\
\text { Procesos } \\
\text { cognitivos } \\
\text { involucrados: } \\
\text { Análisis, síntesis } \\
\text { y pensamiento } \\
\text { crítico }\end{array}$ \\
\hline
\end{tabular}


Bio - grafía. Escritos sobre la Biología y su Enseñanza. ISSN 2027-1034

Edición Extraordinaria. p.p. 1533 - 1540

Memorias del IX Encuentro Nacional de Experiencias en Enseñanza de la Biología y la Educación Ambiental. IV Congreso Nacional de Investigación en Enseñanza de la Biología.

\begin{tabular}{|l|l|l|}
\hline & $\begin{array}{l}\text { Consecuencias que se derivaron en la solución } \\
\text { planteada para cada uno de los actores } \\
\text { involucrados. }\end{array}$ & $\begin{array}{l}\text { Subfase de propuestas para la toma de decisiones } \\
\text { Finalmente, el equipo plantea otra alternativa de } \\
\text { solución, que incluirá la valoración de sus efectos } \\
\text { para los actores en el contexto de estudio. }\end{array}$ \\
\hline
\end{tabular}

Nota: Landazábal y Gamboa (en prensa). El proceso de argumentación en la formación inicial de docentes: una experiencia mediada por Dígalo y Simas. Bogotá: Universidad Distrital Francisco José de Caldas.

El estudio de caso como estrategia didáctica

\begin{tabular}{l|ll}
\hline \multicolumn{1}{c|}{ Retos } & \multicolumn{3}{|c}{ Oportunidades } \\
\hline $\begin{array}{l}\text { Articular la estrategia didáctica a una } \\
\text { estrategia pedagógica que promueva el } \\
\text { desarrollo humano. }\end{array}$ & $\begin{array}{l}\text { Fomentar el trabajo colaborativo y } \\
\text { cooperativo con base en el trabajo en } \\
\text { equipo. }\end{array}$ \\
\hline $\begin{array}{l}\text { Definir casos pertinentes que generen } \\
\text { debate y permitan la construcción de } \\
\text { conocimiento. }\end{array}$ & $\begin{array}{l}\text { Fomentar la cultura participativa como } \\
\text { elemento de responsabilidad social. }\end{array}$ \\
\hline $\begin{array}{l}\text { Describir las situaciones involucradas en } \\
\text { el caso para la comprensión. }\end{array}$ & Formular hipótesis para el debate. \\
\hline $\begin{array}{l}\text { Platear alternativas de solución para el } \\
\text { caso con el fin de disminuir el impacto }\end{array}$ & $\begin{array}{l}\text { Analizar los conceptos teórico para la } \\
\text { comprensión de la situación } \\
\text { sobre los actores involucrados. }\end{array}$ \\
\hline $\begin{array}{l}\text { Tomar decisiones acertadas para } \\
\text { disminuir impactos sobre el ambiente y los } \\
\text { actores. }\end{array}$ & $\begin{array}{l}\text { Reconocer y localizar información } \\
\text { requerida para resolver el caso planteado. }\end{array}$ \\
\hline $\begin{array}{l}\text { Definir etapas para el desarrollo del } \\
\text { pensamiento dialógico crítico con base en } \\
\text { las actuaciones de los actores para la la } \\
\text { resolución del caso. }\end{array}$ & $\begin{array}{l}\text { Reflexionar sobre las decisiones tomas y y } \\
\text { sus implicaciones. }\end{array}$ \\
\hline $\begin{array}{l}\text { Promover la motivación de los } \\
\text { participantes para lograr los objetivos } \\
\text { propuestos. }\end{array}$ & $\begin{array}{l}\text { Fomentar el pensamiento dialógico crítico } \\
\text { para la toma de decisiones. }\end{array}$ \\
\hline $\begin{array}{l}\text { Seleccionar de manera pertinente el caso } \\
\text { en la fase que se requiere para el logro de }\end{array}$ & $\begin{array}{l}\text { Plantear casos por niveles de acuerdo a } \\
\text { los objetivos de formación }\end{array}$ \\
los objetivos.
\end{tabular}

\section{PROPÓSTOS}

Reflexionar acerca de las concepciones que sobre pedagogía y didáctica tienen los docentes participantes y la implicación de ellas en la construcción de un ambiente de enseñanza-aprendizaje de la biología desde la planeación. 
Bio - grafía. Escritos sobre la Biología y su Enseñanza. ISSN 2027-1034

Edición Extraordinaria. p.p. 1533 - 1540

Memorias del IX Encuentro Nacional de Experiencias en Enseñanza de la Biología y la Educación Ambiental. IV Congreso Nacional de Investigación en Enseñanza de la Biología.

Vivenciar un ambiente de enseñanza-aprendizaje la enseñanza de la biología desde el reconocimiento de los actores y los factores intervinientes para el logro de los objetivos propuestos.

Adquirir competencia para la selección e implementación adecuada de casos en espacios educativos en respuesta a la responsabilidad personal y social del docente en el proceso de enseñanza-aprendizaje, asumiendo el estudio de ellos, como una estrategia didáctica para la construcción de conocimientos.

\section{ASPECTOS PROCEDIMENT ALES}

El taller tiene una duración de dos horas, las cuales involucran a los participantes en la realización de una serie de actividades que configuran la secuencia descrita a continuación:

(C) Fase Inicial - Reconocimiento de los actores en un proceso de enseñanzaaprendizaje: 15 minutos. Presentación de los participantes en el taller y socialización de los intereses que motivaron su participación en la actividad.

(C) Fase Intermedia - Identificación de las denominaciones de los casos que se pueden proponer como estrategia didáctica en un ambiente de enseñanzaaprendizaje de la biología. 60 minutos.

Caso centrado en las descripciones. Fase de Identificación. Vídeo: Cambiar la educación para cambiar el mundo. Duración en tiempo 1:30. Autor: David Berruezo Varela. 15 minutos para consolidar y responder al interrogante.

¿La educación es un proceso que se suscita netamente en el aula?

Trabajo en grupo: reportar elementos descriptivos, situaciones descritas y sentido de la información, hipótesis frente a la situación, conceptos teóricos para la comprensión del tema en el cual se centra la situación.

Caso centrado en el análisis crítico de toma de decisiones. Fase de toma de decisiones. Vídeo: 10 fotografías que demuestran que la tierra está muriendo. Duración en tiempo 3:01. Autor Top View Viral. Complemento video: La tierra está muriendo. Duración en tiempo 8:47. Autor: Dankeu. 20 minutos para consolidar y responder al interrogante

¿Cómo puede el Licenciado en Biología transformar la situación actual del planeta?

Caso centrado en la resolución de problemas. Fase de propuestas. Con base en el material entregado por grupo. Resolver el problema contextualizado. 25 minutos para consolidar y responder frente al caso presentado. Involucra identificar las situaciones problema, jerarquizarlas, analizar la toma de decisiones en el caso y la incidencia entre 
Bio - grafía. Escritos sobre la Biología y su Enseñanza. ISSN 2027-1034

Edición Extraordinaria. p.p. 1533 - 1540

Memorias del IX Encuentro Nacional de Experiencias en Enseñanza de la Biología y la Educación Ambiental. IV Congreso Nacional de Investigación en Enseñanza de la Biología.

los actores y finalmente, el grupo planteará una alternativa de solución que mitigue el impacto sobre el medio y los actores.

(C) Fase Final - Socialización de las experiencias del taller y conclusiones. 30 minutos.

$\checkmark$ Materiales requeridos: Marcadores de colores, (1) Un marcador permanente por participante, un paquete de fichas bibliográficas y papel periódico para cada grupo.

\section{BIBLIOGRAFÍA}

Aubert, A., Flecha, A., García, C., Flecha, R., Racionero, S. (2013) Aprendizaje Dialógico en la Sociedad de la Información ( $3^{\mathrm{a}}$ ed.), Barcelona: Hipatia.

Álvarez, C., González, L., y Larrinaga, A. (2013). Aprendizaje dialógico: una apuesta de centro educativo para la inclusión. TABANQUE Revista pedagógica, 26, p 209-224. Universidad de Valladolid. Recuperado de https://dialnet.unirioja.es/descarga/articulo/4754777.pd

Castro, C. (2014). El Método de casos como estrategia de enseñanza-aprendizaje. Cada acto educativo es un acto ético. Cuernavaca: Universidad Autónoma del Estado de Morelos.

Díaz-Alcaraz, F. (2002). Didáctica y Currículo: un enfoque constructivista. Cuenca: Ediciones Universidad de Castilla-La Mancha.

Díaz Barriga, Frida. (2005). Enseñanza situada: Vínculo entre la escuela y la vida. México: McGraw Hill.

Instituto tecnológico de Monterrey. (s.f.). El estudio de caso como técnica didáctica. México: Instituto tecnológico de Monterrey. Recuperado de http://sitios.itesm.mx/va/dide2/documentos/casos.PDF

Landazábal y Gamboa (en prensa). El proceso de argumentación en la formación inicial de docentes: una experiencia mediada por Dígalo y Simas. Bogotá: Universidad Distrital Francisco José de Caldas.

Mansilla, J., y Beltrán, J. (2013). Coherencia entre las estrategias didácticas y las creencias curriculares de los docentes de segundo ciclo, a partir de las actividades didácticas. Perfiles Educativos. XXXV, (139), p.25-39.

Tobón, S. (2006). Aspectos básicos de la formación basada en competencias. Talca: Proyecto Mesesup. Recuperado de http://www.urosario.edu.co/CGTIC/Documentos/aspectos basicos formacion bas ada competencias.pdf

Valdes, P. y Valdes, R. (2004). Tres ideas básicas de la didáctica de las ciencias. En: Didáctica de las Ciencias. Nuevas Perspectivas. La Habana: Pueblo y Educación. Recuperado http://www.unesco.org/fileadmin/MULTIMEDIA/FIELD/Havana/images/didaticasdel asciencias2004.pdf

Zambrano, A. (2002). Los hilos de la palabra: pedagogía y didáctica. Santiago de Cali: Nueva Biblioteca Pedagógica. 